\title{
The ATLAS Beam Conditions and Beam Loss Monitors
}

\author{
Boštjan Maček* on behalf of ATLAS BCM and BLM group \\ Jožef Stefan Institute, Ljubljana \\ E-mail: bostjan.macek@ijs.si
}

The ATLAS Beam Conditions and Beam Loss Monitors are designed to detect early signs of beam instabilities and protect the ATLAS Inner Detector silicon trackers against damaging LHC beam incidents by initiating beam abort in case of beam failures. Polycrystalline Chemical Vapour Deposition (pCVD) diamond was chosen as the sensor material for both systems. Beam Conditions Monitor (BCM) will provide real-time monitoring of instantaneous particle rates close to the interaction point (IP) of ATLAS detector. There are four detector modules on each side of IP. Each of them is built of two pCVD diamond pad sensors read out in parallel. Using fast front-end and signal processing electronics the time-of-flight and pulse amplitude measurements will be performed to distinguish between normal collisions and background events due to natural or accidental beam losses. Additionally, BCM will also provide coarse luminosity information. Second system, the ATLAS Beam Loss Monitor (BLM), is an independent system which was recently added to complement the BCM. It is a current measuring system that was partially adopted from the BLM system developed by the LHC beam instrumentation group with pCVD diamond pad sensors replacing ionization chambers.

9th International Conference on Large Scale Applications and Radiation Hardness of Semiconductor Detectors

30 September - 2 October 2009

Florence, Italy

\footnotetext{
*Speaker.
} 


\section{Introduction}

Potential abnormal beam conditions, resulting from LHC equipment failure, could cause substantial damage to the detectors. To that end, the experiments decided to develop their own beamsafety systems in parallel to the ones provided by Large Hadron Collider (LHC). In ATLAS spectrometer this role has been given to the Beam Conditions Monitor (BCM) and Beam Loss Monitor (BLM) systems. Their main purpose is early detection of beam instabilities and, if needed, initiation of the beam abort. Additionally ATLAS BCM will also provide a coarse relative luminosity measurement, also on a bunch by bunch basis, as a complementary information to LUCID, ATLAS' main luminosity monitor.

\section{ATLAS BCM}

The ATLAS BCM consists of two sets of four modules - one on each side of the interaction point, at $z_{B C M}= \pm 1.84 \mathrm{~m}$. This symmetrical positioning is shown in figure 1 , where also the basic principle of operation is sketched. Utilizing the time of flight measurement for each of these two stations, and looking at the time difference, one can obtain the position of the particle source. Particles created in bunch crossings at interaction point are detected on both sides in coincidence at 6.25 ns after the collision. These are called in-time hits. In contrast, the particles originating upstream, at $|z|>\left|z_{B C M}\right|$, hit the nearest detector station $6.25 \mathrm{~ns}$ before the actual bunch crossing (out-of-time hits), thus $12.5 \mathrm{~ns}$ before they hit the opposite side of BCM. This time difference is almost exactly half of the time interval between the two consecutive LHC bunch crossings. Given sufficient timing resolution, in-time hits can be used to monitor collision conditions - luminosity, and the out-of-time hits can be a useful selection tool to identify background events. Short description of the system is provided in the following section, for details see [1], [2].

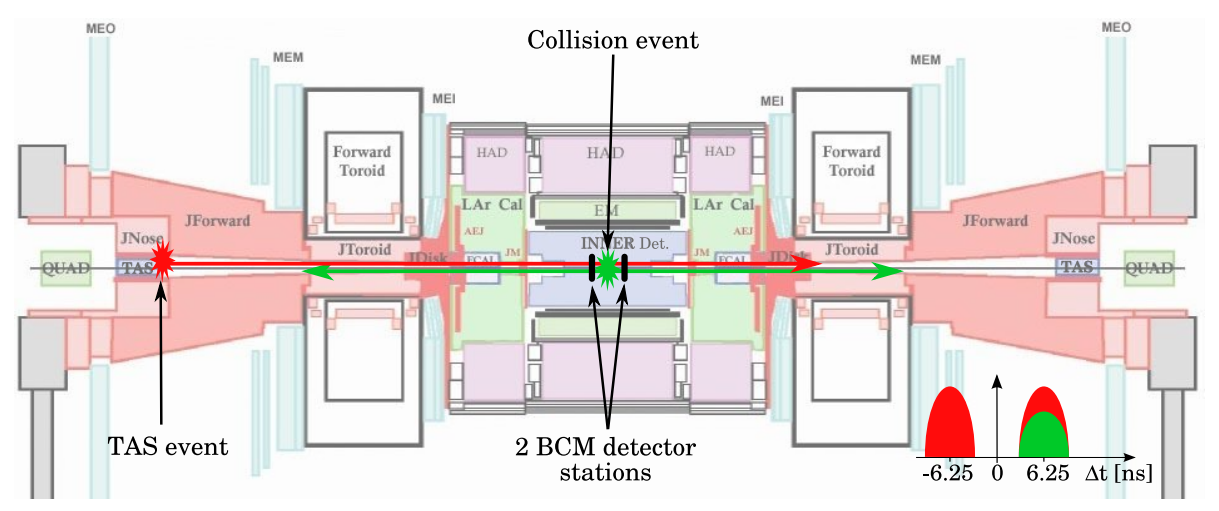

Figure 1: Principle of operation of Beam Condition Monitor. In case of the desired proton-proton interaction at interaction point (green) secondary particles reach both sides of BCM system simultaneously. In the case of anomalous event such as proton hitting the TAS collimator (red) one side of BCM system detects secondary particles before the other. The $\left|z_{B C M}\right|= \pm 1.84 \mathrm{~m}$ puts the time difference between the two sides at $\sim 12.5 \mathrm{~ns}$. 


\subsection{Detector modules}

All eight modules are mounted at radius of $55 \mathrm{~mm}$ from the beam. Given their distance from the interaction point this puts them in the very forward region, thus they reside in a very harsh radiation environment. The expected ionization doses are about $0.5 \mathrm{MGy}$ and fluencies of about $10^{15}$ particles $/ \mathrm{cm}^{2}$ in ten years of ATLAS operation at nominal LHC luminosity. To satisfy these requirements $\mathrm{pCVD}$ diamond was chosen as the sensor material, for its radiation hardness and fast signal response (rise time $<1 \mathrm{~ns}$, signal width $\sim 2 \mathrm{~ns}$ ) allows the measurement on a bunch by bunch basis. Their additional big asset is that they draw negligible leakage currents, eliminating the need for active cooling. Polycrystalline diamonds are of $1 \mathrm{~cm} \times 1 \mathrm{~cm}$ size and $500 \mu \mathrm{m}$ thickness. They were developed by a collaboration of RD42 [3] and Element Six Ltd. [4], and were proven to be radiation hard and to produce fast signals. To achieve high and narrow signal pulses the sensors are operated close to the charge carrier saturation velocity, at a bias voltage of $\pm 1000 \mathrm{~V}$. In order to optimize the detector response two such diamonds are connected in parallel with ceramic insert in-between. Such a configuration doubles the signal while increasing the noise by only $30 \%$. Bias voltage is applied to outside surfaces of this 'double-decker' configuration, while the inner ones are connected to ground and treated as signal lines, leading to the front-end electronics within the module. There are two current amplifiers: $500 \mathrm{MHz}$ Agilent MGA-62563 GaAs MMIC and $1 \mathrm{GHz}$ Mini Circuits Gali 52 HBT chips, each providing an amplification of about $20 \mathrm{db}$. The module is shown in figure 2 . In addition the expected anisotropy of particle direction is exploited by mounting the modules at $45^{\circ}$ with respect to the most probable particle direction along the beam line to gain an additional factor of $\sqrt{2}$ in the signal.

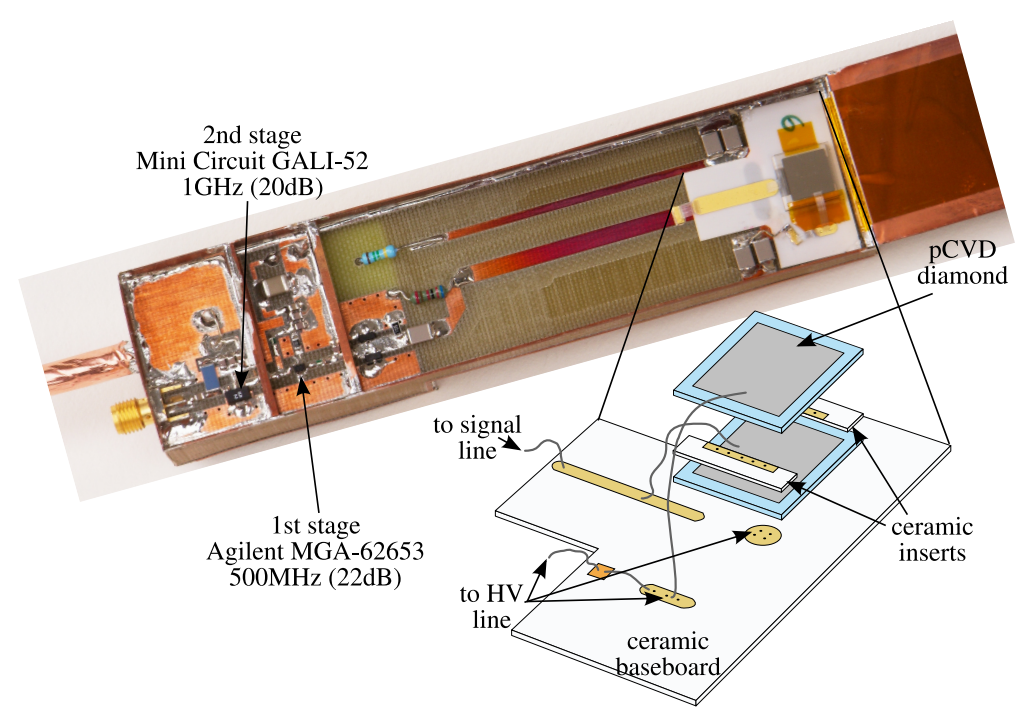

Figure 2: BCM module without cover, showing the pCVD diamond and the two front-end amplifiers.

\subsection{Read-out}

Signals from the modules are routed through $15 \mathrm{~m}$ of coaxial cables to the region close to ATLAS toroid magnets where much lower levels of radiation are expected (approx. 10Gy), where 
the signals are digitized. The digitization circuit hosts a fourth-order low-pass filter with $300 \mathrm{MHz}$ bandwidth, NINO chip [5], and laser diodes. The input filter increases the signal to noise ratio and splits the signal in ratio $1: 10$ in order to increase dynamical range of the detector. These two signals are than passed to the NINO chip, originally designed for time of flight measurement in ALICE RPC detector. It features a differential input amplifier, discriminator and time over threshold measurement. In the last step, the NINO time-over-threshold output signal is converted to an optical signal, using radiation tolerant laser diodes, and then transmitted over $70 \mathrm{~m}$ of optical fibers to the ATLAS counting room, to be received by photo diodes and converted to PECL electric level signals that get processed by back-end electronics. For this part the BCM uses two Xilinx ML410 development boards, each hosting one Vitrex-4 FPGA chip. Each has 8 dedicated high speed RocketIO input sampling channels, that operate at $2.56 \mathrm{GHz}$ frequency, thus giving $\sim 400 \mathrm{ps}$ timing resolution. Here data is interpreted as in-time or out-of-time hits, processed, stored, and further dispatched to different ATLAS and LHC services:

- LHC Beam/Injection Interlock/Permit System. One signal per FPGA is used by the BCM. Each of this signals can prohibit injection of proton bunches into LHC and a coincidence can initiate a dump of the circulating beam in the machine.

- ATLAS Detector Safety System is a hardware interlock system, offering the ATLAS detector components possibility to react to unwanted situations and protect the hardware. BCM provides four signals; two with severity of warning and two of error.

- ATLAS Detector Control System is a slow detector control system that resides in the software world. Though slower than DSS it provides valuable control since is enables more sophisticated decision making and greater flexibility.

- Central Trigger Processor, as the main ATLAS information filter, receives 6 bits of Level1 trigger information from BCM.

- ATLAS Trigger-DAQ, receives a data fragment also from BCM and incorporates it into the event if passed through all trigger levels. Two arrival times and two time-over-threshold measurements can be recorded in each bunch-crossing for each channel, and in each event data for 31 bunch-crossings(BC) is written.

\subsection{Commissioning results with cosmic data}

Towards the end of year 2008 and in the beginning of 2009 ATLAS Inner Detector recorded combined cosmic data with two different triggers. One was the trigger issued by Resistive Plate muon Chambers (RPC) [6], and the other by the fast-OR mechanism of Transition Radiation Tracker (TRT) [7]. As described above, for each Level 1 Accept signal, data for 31 bunch crossings was recorded. Afterwards the analysis reconstructed hits, and histogrammed the number of bunch crossings where these hits were reconstructed. Figure 3 shows the distributions for RPC and TRT trigger samples. Both timing plots exhibit a Gaussian in-time peak with an underlying flat random background. The TRT plot has narrower peak width, which can be explained by the lower jitter of this trigger. Extracted fit parameters show that 1 million TRT triggered events resulted in $\sim 9$ 
BCM hits from cosmic rays and 10 million RPC triggers were required to get 9 such BCM hits. The estimated probability for a noise hit in the BCM in one $\mathrm{BC}$ is around $10^{7}$.
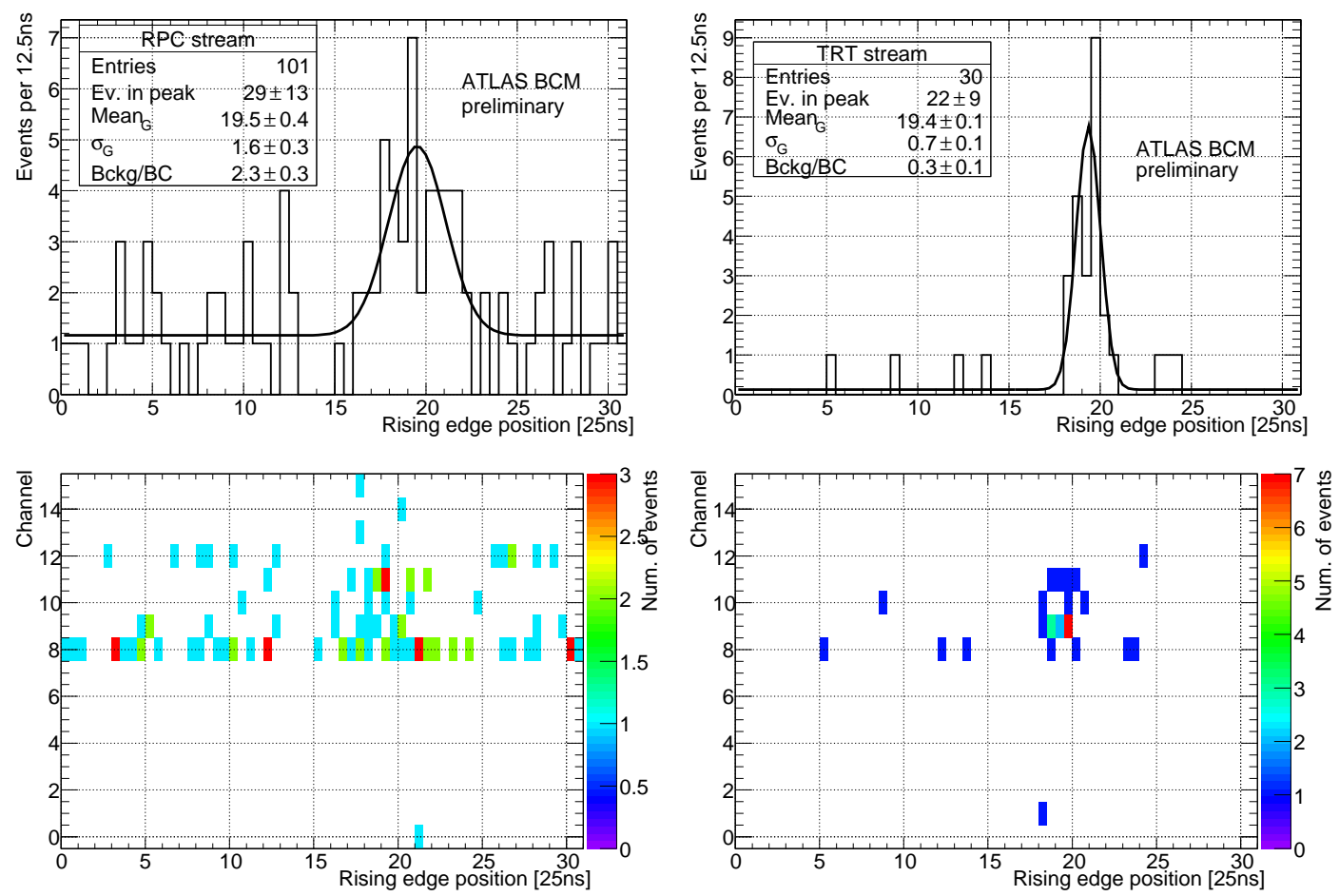

Figure 3: Timing distribution for RPC and TRT triggered BCM hits collected during ATLAS cosmic data taking. The time scale is in units of BCs. The bottom two plots show the same timing distribution per channel.

BCM also participated in the June 2009 ATLAS cosmic data taking. The timing plots for the random and IDCosmic triggered events are shown in figure 4. The IDCosmic trigger selected events that gave a track in ATLAS Inner Detector at trigger Level2. Analysis shows that 1 million IDCosmic triggers gave around 6 genuine BCM cosmic ray hits. The probability for a noise hit in one $\mathrm{BC}$ was found to be 78 times higher than in 2008 Cosmic run due to the lower threshold settings in 2009 run.

\subsection{Luminosity}

Beside the basic functionality of protecting ATLAS, BCM is capable of providing luminosity monitoring. Symmetrical layout helps once again to distinguish between background and collisions. With measuring the rate of coincidences (in-time hits on both sides) one can drastically reduce the background. The relation between the coincidence rate and luminosity is non-linear though, but can be modeled with two parameters: detector efficiency and coincidence probability per single proton-proton collision. These parameters can be extracted from Monte-Carlo simulation or from dedicated low luminosity runs of LHC with the average number of interactions per bunch crossing much smaller than unity. Once calibrated this method offers good sensitivity over wide range of luminosities even when simplified to simple event counting. In addition to instantaneous 

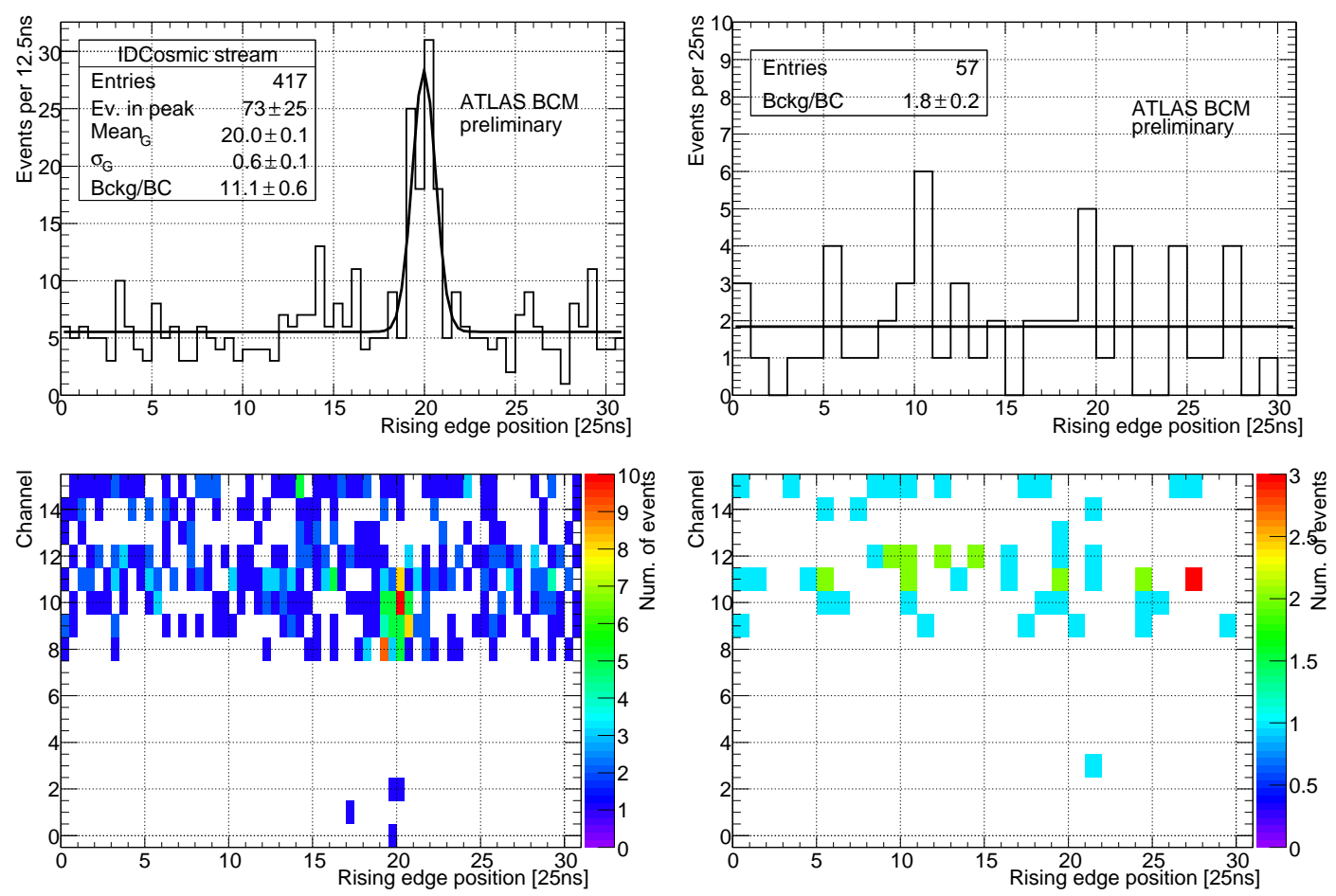

Figure 4: Timing distribution for IDCosmic and random triggered BCM hits collected during ATLAS cosmic data taking. The time scale is in units of BCs. The bottom two plots show the same timing distribution per channel.

luminosity (short time period sum over all bunches) the BCM will also provide bunch by bunch luminosity measurement at the end of each luminosity block.

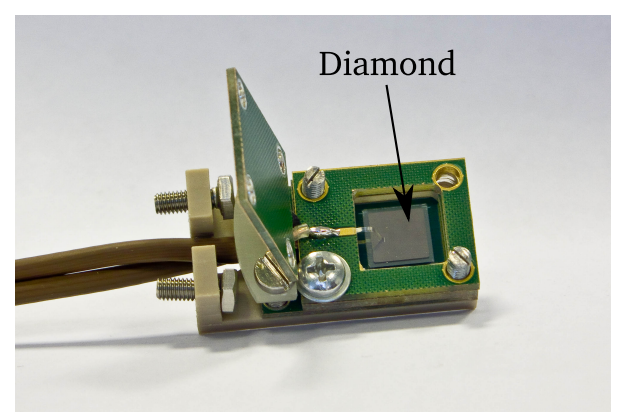

Figure 5: One of twelve BLM modules before installation. Diamond is inside G10 casing and the assembly is mounted on a peek holder.

\section{ATLAS BLM}

The ATLAS Beam Loss Monitor (BLM) is constructed as an independent system and has been recently added as a backup to BCM. The detectors themselves are again based on pCVD 
diamond sensors, $8 \mathrm{~mm} \times 8 \mathrm{~mm}$ in size, and $500 \mu \mathrm{m}$ thick. Each diamond is placed in a module box constructed from G10 plates offering electrical shielding and mechanical support (see figure 5). There are 6 modules on each side of the detector, mounted on the Inner Detector End Plate at $z_{B L M}= \pm 3.45 \mathrm{~m}$ and radius of $6.5 \mathrm{~cm}$. The radiation induced current in each module is read out by the BLM-CFC card which translates this current to a digital signal that is sent via an optical link to ATLAS USA15 counting room. Here BLMTC VME based board integrates these signals over different time periods ranging from $40 \mu \mathrm{s}$ to $84 \mathrm{~s}$. If two of these readings, corresponding to the same side of the detector, exceed a predefined threshold, a beam dump request will be issued.

\section{References}

[1] W. Trischuk et al., JINST, 3, P02004 (2008).

[2] I. Dolenc, Development of Beam conditions monitor for ATLAS experiment, Ph.D. thesis, University of Ljubljana, 2008.

[3] CERN RD-42 Collaboration: CVD Diamond Radiation Detector Development (http://rd42.web.cern.ch/RD42/).

[4] Element Six Ltd., Kings Ride Park, Ascot, Berkshire SL5 8BP, UK.

[5] F. Anghinolfi, et al., NINO: an ultra-fast and low-power front-end amplifier and discriminator ASIC for the multi-gap resistive plate chambers, Nucl. Instr. and Meth. A 533 (2004) 183-187.

[6] The ATLAS collaboration, The ATLAS Experiment at the CERN Large Hadron Collider, JINST 3, 1-422 (2008).

[7] The TRT Fast-OR Trigger, to be published as ATLAS note. 